\title{
Is Middle-Upper Arm Circumference "normally" distributed? Secondary data analysis of 852 nutrition surveys
}

\author{
Severine Frison ${ }^{1 *}$, Francesco Checchi $^{3}$, Marko Kerac ${ }^{1}$ and Jennifer Nicholas ${ }^{2}$
}

\begin{abstract}
Background: Wasting is a major public health issue throughout the developing world. Out of the 6.9 million estimated deaths among children under five annually, over 800,000 deaths (11.6\%) are attributed to wasting. Wasting is quantified as low Weight-For-Height (WFH) and/or low Mid-Upper Arm Circumference (MUAC) (since 2005). Many statistical procedures are based on the assumption that the data used are normally distributed. Analyses have been conducted on the distribution of WFH but there are no equivalent studies on the distribution of MUAC.

Methods: This secondary data analysis assesses the normality of the MUAC distributions of 852 nutrition crosssectional survey datasets of children from 6 to 59 months old and examines different approaches to normalise "nonnormal" distributions.

Results: The distribution of MUAC showed no departure from a normal distribution in 319 (37.7 \%) distributions using the Shapiro-Wilk test. Out of the 533 surveys showing departure from a normal distribution, 183 (34.3\%) were skewed (D'Agostino test) and 196 (36.8\%) had a kurtosis different to the one observed in the normal distribution (Anscombe-Glynn test). Testing for normality can be sensitive to data quality, design effect and sample size. Out of the 533 surveys showing departure from a normal distribution, 294 (55.2\%) showed high digit preference, 164 (30.8\%) had a large design effect, and 204 (38.3 \%) a large sample size. Spline and LOESS smoothing techniques were explored and both techniques work well. After Spline smoothing, $56.7 \%$ of the MUAC distributions showing departure from normality were "normalised" and $59.7 \%$ after LOESS. Box-Cox power transformation had similar results on distributions showing departure from normality with $57 \%$ of distributions approximating "normal" after transformation. Applying Box-Cox transformation after Spline or Loess smoothing techniques increased that proportion to 82.4 and $82.7 \%$ respectively.
\end{abstract}

Conclusion: This suggests that statistical approaches relying on the normal distribution assumption can be successfully applied to MUAC. In light of this promising finding, further research is ongoing to evaluate the performance of a normal distribution based approach to estimating the prevalence of wasting using MUAC.

Keywords: Normal distribution, Middle-Upper Arm Circumference, Child malnutrition, Wasting, Probit

\section{Background}

Wasting is a major public health issue throughout the developing world. The United Nations Children's Fund's (UNICEF) latest report on the State of the World's Children [1] estimates that $10 \%$ of children under 5 years

\footnotetext{
*Correspondence: severine.frison@gmail.com

${ }^{1}$ Department of Population Health, London School of Hygiene and Tropical Medicine, Keppel Street, London WC1E 7HT, UK

Full list of author information is available at the end of the article
}

old in least developed countries are wasted. Out of the 6.9 million estimated deaths among children under five annually, over 800,000 deaths $(12.6 \%)$ are attributed to wasting [2]. Wasting is quantified as Weight-For-Height (WFH) $<-2$ standard deviations (SD) from the World Health Organization (WHO) reference median and/or Mid-Upper Arm Circumference (MUAC) $<125 \mathrm{~mm}$. MUAC has been adopted by the World Health Organisation (WHO) as a measure of wasting and is increasingly 
recognised as a very useful measure of anthropometric status $[3,4]$.

Many statistical procedures are based on the assumption that the data follow a normal distribution. The shape of the normal distribution (the characteristic "bell curve") is quantified by two parameters: the mean and the standard deviation, and follows important properties: (1) it is always symmetrical with equal areas on both sides of the curve; (2) the highest point on the curve corresponds to the mean which equals the median and the mode; (3) the spread of the curve is determined by the standard deviation; and (4) as with all probability density functions the area under the curve must sum to the total probability of 1 [5]. The distribution of many characteristics in nature is normal or follows some form that can be derived from the normal distribution and specific statistical approaches are based on the properties of a normal distribution. For example, the probit approach $[5,6]$ estimates the prevalence of wasting as the cumulative probability of lying below the relevant MUAC cut-point based on the mean and standard deviation (SD) of the observed data $[5,6]$.

There are graphical and statistical methods for evaluating normality. Graphical methods include histograms and normality plots. Statistical methods include diagnostic hypothesis tests for normality, and a normal distribution has a skewness of 0 and kurtosis of $3[7,8]$. Skewness is a measure of the asymmetry of a distribution around its mean while Kurtosis indicates heavy tails and "peakedness" relative to a normal distribution $[9,10]$. The ability to detect departure from a normal distribution can be sensitive to local peaks and troughs in the distribution. A way to deal successfully with this issue is to apply smoothing techniques (fit a smooth curve to a set of noisy observations) using different methods such Spline function or Locally Weighted Scatterplot Smoothing (LOESS) [11-13]. For distribution originating from cluster surveys, it may be expected that high clustering in observations (large design effect) lead to asymmetric distributions, e.g. featuring a long tail of low MUAC observations. When a variable is not normally distributed for a reason other than the ones above, it can often be transformed and tested for normality using power transformations such as the Box-Cox transformation $[14,15]$.

Although the violation of the normal distribution assumption often increases chances of committing either a type I or II error, very few researchers test whether the assumption does indeed hold before carrying out statistical analyses [16, 17]. Previous studies have assessed the distribution of WFH [18-20] but there are no equivalent studies on the distribution of MUAC. This paper assesses the normality of the MUAC distribution graphically and statistically, and explores different transformations and smoothing techniques in order to reach normality. Findings presented pertain to a broader project to develop a more efficient method for estimating the prevalence of wasting using MUAC as the primary index, which relies heavily on MUAC distributions meeting normality criteria.

\section{Methods}

\section{Study design and inclusion criteria}

A total of 1068 cross-sectional survey datasets from various settings were shared by six organisations (UNICEF, Food Security and Nutrition Analysis Unit, Epicentre/ Médecins Sans Frontières, Action Against Hunger, Concern Worldwide and Goal). The study size depended on availability of surveys and on specific inclusion criteria. Eligible datasets had to: (1) include MUAC, oedema, age, weight and height as well as meta-data on country, livelihood, residence, cluster (if cluster surveys) and date; (2) have a minimum of 25 clusters if cluster surveys [21, 22]. The last criteria aimed to minimise selection bias, as surveys with a small number of clusters may not be representative of the population. The surveys were exhaustive or clustered surveys. The datasets were cleaned and records with extreme or missing values were excluded: Children were excluded if any of the following data were missing: age; sex; height; weight; MUAC; oedema. Those with highly improbable extreme values ('flags') were also excluded from analysis: MUAC $<85 \mathrm{~mm}$ or MUAC $>200 \mathrm{~mm}$, age $<6$ months or age $>59$ months, Weight-For-Age (WFA) $<-6.0$ SD or WFA $>+5.0 \mathrm{SD}$, Height-For-Age $(\mathrm{HFA})<-6.0 \mathrm{SD}$ or HFA $>+6.0 \mathrm{SD}$, WFH $<-5.0$ SD or WFH $>+5.0$ SD (WHO "flags" were applied on SD for WFH, WFA and HFA [23]).

\section{Database}

Out of the 1068 surveys collected, 852 surveys were included in the secondary data analysis (55 exhaustive surveys and 797 clustered surveys). The 852 surveys contained 668,975 children of which 25,134 (3.76\%) presented highly improbable values and were excluded from the analysis. The database included six variables for anthropometry (sex, MUAC, oedema, age, weight and height), six meta-data variables (organisation, country, livelihood, residence, cluster (when cluster surveys) and date). Other variables were computed for the purpose of this analysis: (1) the normality of the distribution (binary: $1=$ yes $/ 0=$ no using Shapiro-Wilk test), (2) the skewness and Kurtosis of MUAC as continuous and binary (binary: $1=$ yes $/ 0=$ no whether the data was skewed or peaked using D'Agostino and Anscombe-Glynn tests respectively), (3) the design effect of surveys (large over 3) (4) digit preference of MUAC. The digit preference variable was equal to 1 -absolute (0.1-proportion of 
each digit preference). Assuming that the proportion of measurements ending with $0,1,2,3,4,5,6,7,8,9$ should equal $10 \%$ and therefore that the highest score was 1 , the lesser the digit preference, the higher the score. A score equal or over 0.75 corresponded to a low digit preference, and under 0.75 to a high digit preference, and (5) survey size category (large size over 900).

\section{Data analysis}

The normality of the MUAC distributions was assessed graphically looking at histograms of MUAC distributions and Q-Q plots (probability plot, "Q" stands for quantile). $\mathrm{Q}-\mathrm{Q}$ plots show sorted values from the data set against the expected values of the corresponding quantiles from the standard normal distribution. The measure of departure from normality was also investigated statistically through Shapiro-Wilk test as well as the D'Agostino test to assess the skewedness and Anscombe-Glynn test to assess the peakedness of MUAC distributions. For each statistical test, a p value less than 0.05 indicates evidence for departure from a normal distribution.

Different methods were explored to transform nonnormal distributions into normal: (1) Spline smoothing (using a spline function) and LOESS (locally weighted scatterplot smoothing using local polynomial regression fitting) techniques were applied to all distribution showing departure from a normal distribution (ShapiroWilk test). While smoothing the data, three criteria were applied: the mean MUAC and MUAC SD, after backtransformation of the smoothed data must be almost unchanged from the non-smoothed mean and SD (properties of a normal distribution is defined by the mean and the SD), and the Shapiro-Wilk test $p$ value has to exceed 0.05. (2) Box-Cox power transformation was applied to all survey showing departure from a normal distribution, and (3) Box-Cox power transformation was applied on surveys showing departure from "normality" after smoothing techniques had been applied.

Spline smoothing fits a spline with knots at every data point $(\mathrm{x})$ by estimating its parameters minimizing the usual sum of squares plus a roughness penalty $(\lambda)$. If $\lambda \rightarrow 0$ imposes no penalty (very close fit), but the resulting curve could be very noisy as it follows every detail in the data. As $\lambda \rightarrow \infty$ the penalty dominates and the solution converges to the ordinary least square line. LOESS is a fairly direct generalization of traditional least-squares methods for data analysis. It fits a polynomial surface determined by one or more numerical predictors, using local fitting. That is, for the fit at point $x$, the fit is made using points in a neighbourhood of $x$, weighted by their distance from $x$ (with differences in 'parametric' variables being ignored when computing the distance). The size of the neighbourhood is controlled by $\alpha$ (set by span).
The Box-Cox method transforms data into a "normal" shape using parameter $\lambda$ corresponding to different transformations (i.e. $\lambda=1.00$ : no transformation needed; $\lambda=0.50$ : square root transformation $\lambda=0.29$ : for a transforming power between cube and fourth root $\lambda=0.33$ : cube root transformation $\lambda=0.25$ : fourth root transformation $\lambda=0.00$ : natural $\log$ transformation $\lambda=-0.50$ : reciprocal square root transformation $\lambda=-1.00$ : reciprocal (inverse) transformation and so forth). The most appropriate value of $\lambda$ was identified as that which minimised the departure from a normal distribution on the Shapiro-Wilk test.

$\mathrm{R}$ studio and STATA 13 were used for all analyses [24, 25].

Ethics approval for the project was sought and obtained from the Ethics Committee of the London School of Hygiene and Tropical Medicine (LSHTM Ethics reference 6158).

\section{Results}

The distribution of MUAC showed no departure from a normal distribution in $37.4 \%$ (319 out of 852) of the MUAC distributions using the Shapiro-Wilk test. Out of the 533 surveys showing departure from a normal distribution, 183 (34.3\%) were skewed (D'Agostino test), 196 (36.8 \%) had a kurtosis different to the one observed in the normal distribution (Anscombe-Glynn test) and $70(13.1 \%)$ showed both features. The sensitivity of the Shapiro-Wilk test to departure from normality is influenced by the presence of local peaks and troughs in the distribution such as those caused by digit preference (poor data quality), the design effect (high design effect may lead to asymmetric distributions), and sample size (large sample size results in greater power to detect small departures from a normal distribution). Out of the 533 surveys showing departure from normal distribution, $294(55.2 \%)$ showed high digit preference (score < 0.75$)$ $164(30.8 \%)$ had a large design effect (over 3), and 204 $(38.3 \%)$ a large sample size (>900) (Table 1$)$. The skewness and kurtosis of surveys showing departure from normality included values above and below the value for a normal distribution ( 0 for skewedness and 3 for kurtosis) indicating surveys skewed to right as well as to the left and survey with a distribution flatter or more peaked than the normal distribution (Table 2).

Figure 1 shows examples of distributions of MUAC and their respective $\mathrm{Q}-\mathrm{Q}$ plots for two surveys with very "non-normal" distribution (very low p-value Shapiro-Wilk test) but skewness and kurtosis close to those observed in a normal distribution. Visually neither of the distribution seemed skewed or peaked but digit preferences were visible in both cases which suggest this might be the reason behind the low p-value (Shapiro-Wilk test). 
Table 1 Characteristics of surveys showing departure from a normal distribution (Shapiro-Wilk test, $p<0.05$ ) and effect of transformation and smoothing on specific characteristics $(N=533)$

\begin{tabular}{|c|c|c|c|c|}
\hline \multirow[t]{2}{*}{$\begin{array}{l}\text { Surveys failing Shapiro-Wilk test } \\
(p<0.05) N=533(62.6 \%)\end{array}$} & \multirow[t]{2}{*}{$\mathrm{N}(\%)$} & \multicolumn{3}{|c|}{$\begin{array}{l}\mathrm{N}(\%) \text { with "normal" distribution after transformation or } \\
\text { smoothing }\end{array}$} \\
\hline & & Box-Cox & Spline & Loess \\
\hline All surveys ${ }^{\mathrm{a}}$ & $533(100)$ & $301(56.5)$ & $318(59.7)$ & $304(57.0)$ \\
\hline \multicolumn{5}{|l|}{ By key survey characteristics } \\
\hline Skewed ${ }^{b}$ & $183(34.3)$ & $113(61.7)$ & $81(49.4)$ & $89(48.6)$ \\
\hline Non-normal kurtosis ${ }^{c}$ & $196(36.8)$ & $62(31.6)$ & $137(69.9)$ & $139(70.9)$ \\
\hline Skewed and non-normal kurtosis ${ }^{b, c}$ & $70(13.1)$ & 23(32.9) & $41(58.6)$ & $43(61.4)$ \\
\hline Large design effect (>3) & $164(30.8)$ & $86(52.4)$ & $81(49.4)$ & $92(56.1)$ \\
\hline High digit preference (score $<0.75$ ) & $294(55.2)$ & $143(48.6)$ & $170(57.8)$ & $178(60.6)$ \\
\hline Large sample size $(n>900)$ & $204(38.3)$ & $122(59.8)$ & $95(46.6)$ & $101(49.5)$ \\
\hline
\end{tabular}

${ }^{a}$ Shapiro-Wilk test $(p<0.05) ;{ }^{b} D^{\prime}$ Agostino test $(p<0.05) ;{ }^{c}$ Anscombe-Glynn test $(p<0.05)$

Table 2 Skewness and kurtosis of survey showing departure from a normal distribution $(\mathbf{n}=\mathbf{5 3 3})$

\begin{tabular}{lcccccc}
\hline & Minimum & Lower quartile & Median & Mean & Upper quartile & Maximum \\
\hline Skewness & -0.61 & -0.15 & -0.01 & -0.01 & 0.11 & 0.91 \\
Kurtosis & 2.26 & 2.3 & 3.2 & 3.24 & 3.45 & 5.27 \\
\hline
\end{tabular}

Figure 2 shows examples of distributions of MUAC and their respective Q-Q plots for two surveys with "nonnormal" distribution (low p-value Shapiro-Wilk test) and also skew or kurtosis different to that observed in the normal distribution. The distribution and Q-Q-Plot for the survey shown in panel A has skewed distribution (D'Agostino test) and that shown in panel B has peaked distribution (Anscombe-Glynn test). The distribution in panel A was slightly skewed to the left and there were very visible digit preferences. Peaks are visible in the distribution in panel $\mathrm{B}$ as well as digit preferences.

Table 1 as well as Figs. 1 and 2 suggest the main reason for departure from a normal distribution is due to local peaks and troughs.

\section{Smoothing techniques}

Spline and LOESS smoothing techniques were explored and both techniques work well. After applying Spline smoothing to the distributions showing departure from normality, 301 (56.5\%) of the MUAC distributions showed no departure from normality and 318 (59.7 \%) after LOESS (Table 3).

The average mean MUAC change after Spline smoothing was 0.1 and the mean SD MUAC change was 0.8. All surveys had an average mean MUAC change under 10 and $90 \%$ had a SD change under $10 \%$. After LOESS smoothing, the average mean MUAC change was 0.2 and the average SD MUAC change was 0.9. All surveys had a mean MUAC change under 10 and $84 \%$ had a SD change under $10 \%$.

The effect of Spline and Loess smoothing on "nonnormal" distributions with large design effect, high digit preference, large sample size as well as on skewed distributions and distributions with a kurtosis different from a normal distribution (flat or peaked) was considerable. Approximately half of surveys with large design effect were normalised after Spline and LOESS (49.4 and 56.1\% respectively), about two-third of surveys with high digit preference had a distribution approximating normal after Spline and LOESS (60.6 and $57.8 \%$ respectively), half of surveys with large sample size (46.6 and $49.54 \%$ respectively) as well as half of skewed distributions (49.4 and $48.6 \%$ respectively) and over two-third of surveys with kurtosis different from normal were approximating a normal distribution after Spline and Loess smoothing (69.9 and $70.9 \%$ respectively) (Table 3 ).

\section{Box-Cox power transformation}

Power transformations are typically used to "normalise" skewed distributions. Common power transformations include log, reciprocal, square and square root transformations. After applying the Box-Cox transformation to the 533 distributions showing departure from normality, 304 ( $57 \%$ ) of the distribution were converted to "normal" (Table 3).

The summary statistics of the Box-Cox transformation coefficient [Lambda $(\lambda)$ ] suggest that a variety of different power transformations were required for different surveys and few Lambda values corresponded to common power transformations (Table 4).

The effect of Box-Cox transformation on skewed distributions was sizable with almost two-third of skewed distribution approximation a normal distribution after Bo-Cox 

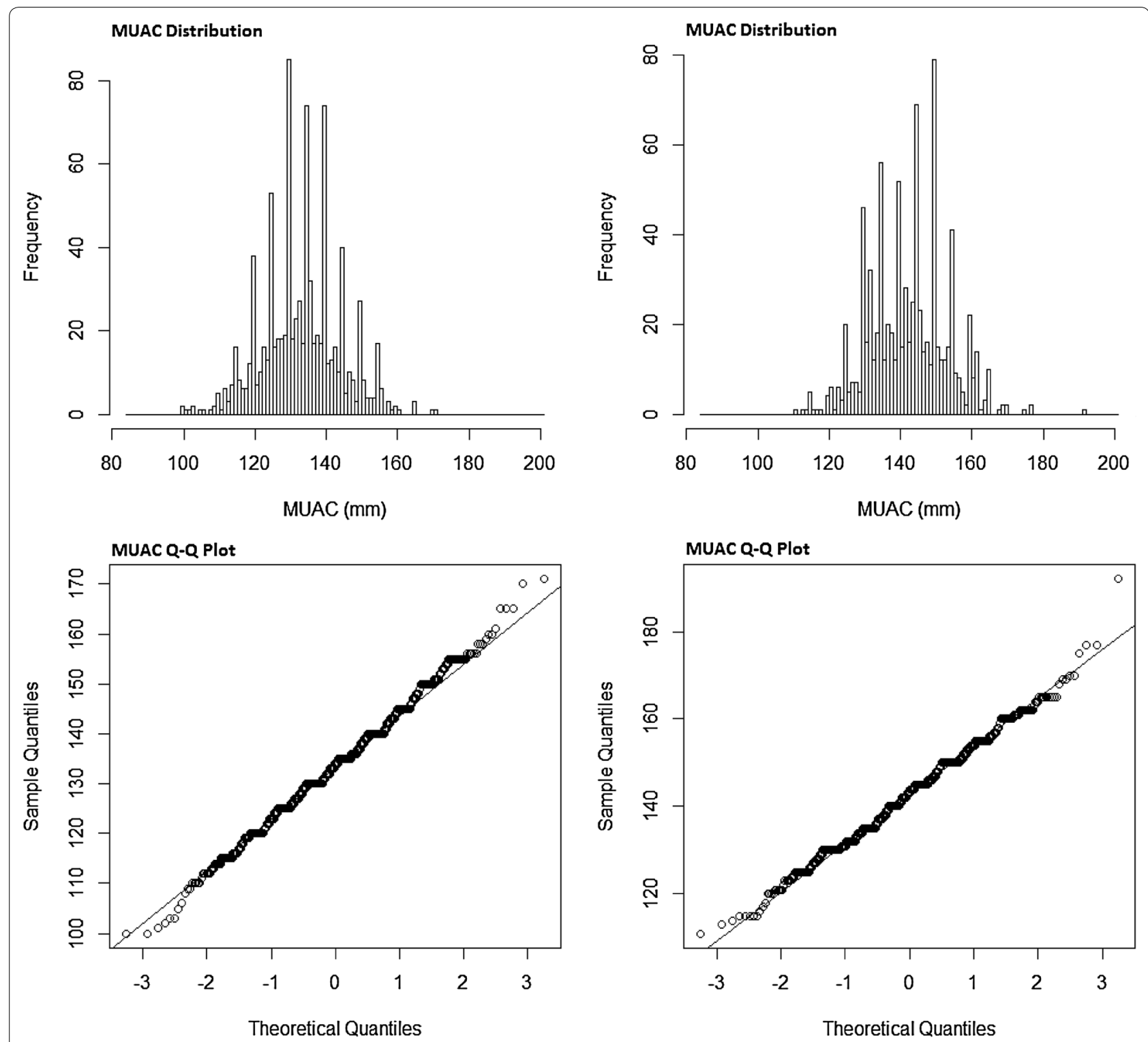

Fig. 1 Examples of non-normal (Shapiro-Wilk test) MUAC distributions and their respective Q-Q plot

transformation $(61.7 \%)$. About half of surveys with large design effect, high digit preference and large sample size distribution were approximating a normal distribution after Box-Cox (52.4, 48.6 and $59.8 \%$ respectively). The effect on distributions with a kurtosis different from normal was less marked with a third (31.6 \%) approximation a normal distribution after Box-Cox transformation (Table 3).

\section{Smoothing and Box-Cox transformation}

Applying Box-Cox transformation on surveys showing departure from a normal distribution after Loess or smoothing techniques increased further the number of "normal" distributions with 401 distributions (82.7\%) after Loess and Box-Cox and 439 (82.4 \%) after Spline and Box-Cox (Table 3).

\section{Discussion}

Over a third of MUAC distributions showed no departure from normality without any transformation and three quarters showed no departure once the data were smoothed or after Box-cox transformation. Applying Box-Cox transformation on surveys showing departure from normality after smoothing resulted in over $80 \%$ of surveys approximating a normal distribution.

Loess smoothing had slightly better outcome then Spline smoothing or Box-Cox transformation alone 

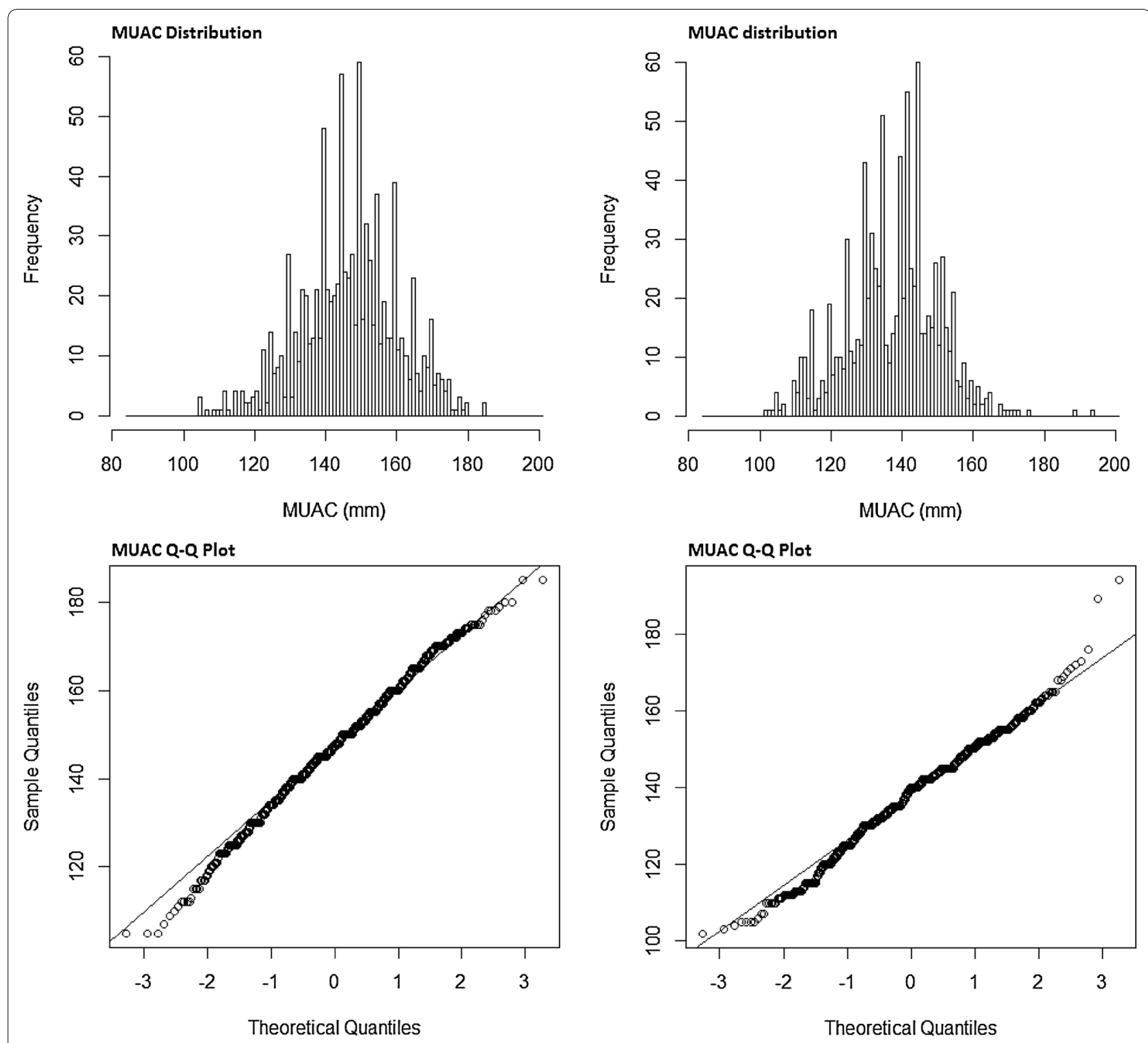

Fig. 2 Examples of a skewed and a peaked distribution and their respective Q-Q plots (D'Agostino and Anscombe-Glynn tests respectively)

Table 3 Smoothing and transformation of surveys showing departure from a normal distribution $(n=533)$

\begin{tabular}{lll}
\hline $\begin{array}{l}\text { Type of transformation or smoothing technique } \\
\text { applied to "non-normal" distributions }(\mathbf{n = 5 3 3 )}\end{array}$ & $\begin{array}{l}\mathbf{N}(\%) \text { "normal” } \\
\text { distributions }\end{array}$ \\
\hline Smoothing & Spline & $301(56.5)$ \\
Lox-Cox transformation & $\begin{array}{l}\text { Power transforma- } \\
\text { tions }\end{array}$ & $318(59.7)$ \\
& Box-Cox after Spline & $439(82.0)$ \\
Smoothing and Box-Cox & Box-Cox after Loess & $441(82.7)$ \\
transformation &
\end{tabular}

in terms of number of distributions approximating a normal distribution but had a change in mean and SD slightly higher (but acceptable) than Spline smoothing. Although Box-Cox transformation performed well, data transformations change the nature of the variable, and any Lambda $(\lambda)$ less than 0.00 has the effect of reversing the order of the data. Even though back transformation restores the data, care should be taken when applying this function [15].

The normality of MUAC distributions is affected by sample size, high digit preference, kurtosis different than 
Table 4 Summary statistics of the Box-Cox transformation coefficient (Lamdba) for surveys showing departure from normality $(n=533)$

\begin{tabular}{lllllll}
\hline & Minimum & Lower quartile & Median & Mean & Upper quartile & Maximum \\
\hline Lambda $(\lambda)$ & -1.2 & 0.61 & 1.08 & 1.03 & 1.51 & 2.73 \\
\hline
\end{tabular}

a normal distribution and skewness. Datasets with larger sample size increase the power of the test to detect small differences when applying normality tests. Digit preference reflects the quality of the data. Training measurers to increase accuracy and precision would decrease digit preference. Both effects were lessened (two-third for digit preference and half for sample size) applying smoothing techniques to the distributions as well as applying Box-Cox transformation (half for both high digit preference and large sample size surveys). Although a third of surveys showing departure from a normal distribution were skewed or had a kurtosis different from a normal distribution, half and over two-thirds (respectively) of these were "normalised" after smoothing. Box-Cox transformation was effective on skewed distributions (almost two-third of skewed distribution "normalised") but didn't perform as well on distributions with a kurtosis different from normal (a third of distributions approximated a normal distribution after Box-Cox).

Few studies have assessed the distribution of WFH. Two looked at the standard deviations of the WFH distributions. In 1977, Waterlow et al. [19]. showed that the WFH distributions were skewed at the upper centiles. Their analysis was performed on data from surveillance or surveys involving nutrition and anthropometry in young children up to the age of 10 years. In 2006, Mei et al. [18] analysed data from 51 DHS surveys representing 34 developing Countries. They found a mean WFH and SD WFH (z-scores) of 0.06 and 1.40 respectively. The mean ranged from -0.91 to 0.83 and the SD range from 1.03 to 1.55 . They concluded that their analysis confirms the $\mathrm{WHO}$ assertion that the SD remains in a relatively small range (i.e. close to SD from a standard normal distribution), no matter the $Z$-score mean although the observed range of SD for was consistently wider. Finally, in 2013, Blanton and Bilukha showed that based on the Shapiro-Wilk test for normality, 6 surveys out of the 10 surveys included in their analysis were "non-normal". All of the surveys had a small amount of skewness ranging from -0.17 and 0.31 as well as a relatively small amount of kurtosis ranging from 0.15 to 0.75 .

Regarding the assessment of MUAC distributions, no equivalent studies were conducted. In 2013, data analysis from 560 cross sectional surveys conducted by Dale et al. [26]. mention the use of Box-Cox transformation to normalise MUAC and WFH data but do not give further details.
There is one main limitation to this study. The database was built based on available small scale surveys that were mainly conducted in areas where there was suspicion of a problem (i.e. high wasting prevalence) compared to national DHS and MICS surveys that are conducted every 3-5 years and show long term trends. However, we do not believe this affects the generalisability of the study. Future research might explore similar analysis on different datasets.

\section{Conclusions}

Over a third of the MUAC distributions of our database were normally distributed. MUAC distributions can easily be normalised applying simple smoothing techniques if the distribution is noisy or displays digit preference and then Box-Cox transformation if indicated (i.e. if data is skewed). This suggests that statistical approaches relying on the normal distribution assumption can be successfully applied to MUAC. In light of this promising finding, further research is ongoing to evaluate the performance of a normal distribution based approach to estimating the prevalence of wasting using MUAC.

\section{Abbreviations}

LOESS: Locally Weighted Scatterplot Smoothing; MUAC: Middle-Upper Arm Circumference; UNICEF: United Nations Children's Fund's; SD: standard deviation; WFH: Weight-For-Height; WHO: World Health Organization.

\section{Authors' contribution}

SF wrote the first draft of the article and had the primary responsibility for the final content. SF was involved in all stages from the conception and design, data acquisition, analysis and interpretation. FC was involved in the conception, design and data acquisition as well as in data analysis and in critically revising different draft versions. JN was involved in data analysis and interpretation as well as in critically revising different draft versions. MK was involved in data interpretation and in critically revising different draft versions. All authors read and approved the final manuscript.

\section{Author details}

${ }^{1}$ Department of Population Health, London School of Hygiene and Tropical Medicine, Keppel Street, London WC1E 7HT, UK. ${ }^{2}$ Department of Medical Statistics, London School of Hygiene and Tropical Medicine, Keppel Street, London WC1E 7HT, UK. ${ }^{3}$ Faculty of Public Health and Policy, LSHTM and Humanitarian Technical Unit, Save the Children, 207 Old Street, London ECIV 9NR, UK.

\section{Acknowledgements}

We would like to thank the following people and organisations for sharing the datasets used for this research: Grainne Moloney and Elijah Odundo from FSNAU, Mara Nyawo from UNICEF Khartoum, Dr. Sheila Isanaka (Nutritional epidemiologist) from Epicentre/MSF Paris, Dr. Benjamin Guesdon and Cécile Salpeteur from Action Against Hunger-Paris, Dr. Anne-Marie Mayer and Gudrun Stallkamp from Concern Worldwide and Claudine Prudhon for sharing data from Goal. We would also like to thank Jane Bruce for supporting the Ph.D. from which this study arose. 


\section{Competing interests}

The authors declare that they have no competing interests.

\section{Funding}

This work was supported by the Office of U.S. Foreign Disaster Assistance (OFDA) and the World Food Programme (WFP) Grant Number ITDCZD07. OFDA and WFP had no role in the design, analysis or writing of this article.

Received: 31 July 2015 Accepted: 19 April 2016

Published online: 04 May 2016

\section{References}

1. UNICEF. The State of the World's Children. New York: UNICEF; 2014

2. Black R, Victora C, Walker S, Bhutta Z, Christian P, De Onis M, Ezzati M, Grantham-Mcgregor S, Katz J, Martorell R, Uauy R. Maternal and child undernutrition and overweight in low-income and middle-income countries. Lancet. 2013;382:427-51.

3. Cluster I-ASCN: Transitioning to the WHO growth standards: implications for emergency nutrition programmes. 2008.

4. Myatt M, Khara T, Collins S. A review of methods to detect cases of severely malnourished children in the community for their admission into community-based therapeutic care programs. Food Nutr Bull. 2006;27:S7-23.

5. Kirkwood BR, Sterne JAC: Essential medical statistics. Blackwell Publishing; 2003.

6. Finney DJ. Probit analysis. Cambridge: Cambridge University Press; 1971

7. Bulmer MG. Principles of statistics. New York: Dover Publications Inc; 1979.

8. DeCarlo LT. On the meaning and use of kurtosis. Psychol Methods. 1997:2:292-307.

9. Thode HC. Testing for normality. New York: Marcel Dekker; 2002.

10. D'Agostino RB, Stephens MA. Goodness-of-fit techniques. New York: Marcel Dekker; 1986.

11. De Boor C. A practical guide to splines (revised edition); 2001.

12. Cleveland WS. Lowess - a program for smoothing scatterplots by robust locally weighted regression. Am Stat. 1981:35:54.
13. Cleveland WS. Robust locally weighted regression and smoothing scatterplots. J Am Stat Assoc. 1979;74:829-36.

14. Carroll RJ, Ruppert D. On prediction and the power transformation family. Biometrika. 1981;68:609-15.

15. Osborne JW. Improving your data transformations: Applying the Box-Cox transformation. In: Improving your data transformations: applying the Box-Cox transformation (editor), vol 15, City:2010.

16. Osborne JW. Notes on the use of data transformations. In: Notes on the use of data transformations. Practical assessment, research and evaluation (editor). City;2002.

17. Altman DG, Martin BJ. Statistics notes: the normal distribution. BMJ. 1995;310:298.

18. Mei Z, Grummer-Strawn LM. Standard deviation of anthropometric Z-scores as a data quality assessment tool using the 2006 WHO growth standards: a cross country analysis. Bull World Health Organ. 2007:85:441-8.

19. Waterlow JC, Buzina R, Keller W, Lane JM, Nichaman MZ, Tanner JM. The presentation and use of height and weight data for comparing the nutritional status of groups of children under the age of 10 years. Bull World Health Organ. 1977;55:489-98.

20. Blanton C, Bilukha O. The PROBIT approach in estimating the prevalence of wasting: revisiting bias and precision. Emerg Themes Epidemiol. 2013;10:8.

21. Binkin N, Sullivan K, Staehling N, Nieburg P. Rapid nutrition surveys: How many clusters are enough? Disasters. 1995;16:99-103.

22. Spiegel PB, Salama P, Maloney S, van der Veen A. Quality of malnutrition assessment surveys conducted during famine in Ethiopia. JAMA. 2004;292:613-8.

23. WHO. 2006. World Health Organization (WHO) Child Growth Standards. http://www.who.int/childgrowth/en/. Accessed 23 June 2015.

24. Software: STATA 13. In: STATA 13 (editor). pp. StataCorp. 2013. Stata Statistical Software: Release 2013. College Station, TX: StataCorp LP.

25. RStudio Team (2015). RStudio: integrated development for R. RStudio, Inc., Boston, MA. http://www.rstudio.com/.

26. Dale NM, Myatt M, Prudhon C, Briend A. Assessment of the PROBIT approach for estimating the prevalence of global, moderate and severe acute malnutrition from population surveys. Public Health Nutr. 2013;16:858-63.

\section{Submit your next manuscript to BioMed Central and we will help you at every step:}

- We accept pre-submission inquiries

- Our selector tool helps you to find the most relevant journal

- We provide round the clock customer support

- Convenient online submission

- Thorough peer review

- Inclusion in PubMed and all major indexing services

- Maximum visibility for your research

Submit your manuscript at www.biomedcentral.com/submit
() Biomed Central 